\title{
Esquema de Alocação Adaptativa de Banda para Tráfego de Redes Utilizando uma Modelagem Multifractal-Fuzzy
}

\author{
Flávio Henrique Teles Vieira, Lee Luan Ling, Flávio Geraldo Coelho Rocha e Scheila Guedes Garcez
}

\begin{abstract}
Resumo-Neste artigo, apresentamos um novo esquema de alocação de banda para tráfego de redes levando em consideração o comportamento multifractal dos fluxos de tráfego. Para tal, funções de base ortonormais e lógica fuzzy são utilizadas, obtendo um modelo híbrido multifractal-fuzzy. Comparações com outros esquemas de alocação de banda comprovam a eficiência do esquema proposto.
\end{abstract}

Palavras-Chave-Alocação de Banda, Tráfego Multifractal, Funções de Base Ortonormais, Predição de Tráfego.

\begin{abstract}
In this paper, we present a new bandwidth allocation scheme for network traffic, taking into account the multifractal behavior of traffic flows. To this end, orthonormal basis functions and fuzzy logic are used, obtaining a multifractal-fuzzy hybrid model. Comparisons with other bandwidth allocation schemes prove the efficiency of the proposed scheme.
\end{abstract}

Index Terms-Bandwidth allocation, Multifractal traffic, Orthonormal basis functions, Traffic prediction.

\section{INTRODUÇÃO}

$\mathrm{Na}$ busca de uma descrição mais completa do tráfego de redes, modelos multifractais têm sido empregados [1] [2]. A modelagem multifractal generaliza os conceitos envolvidos com processos monofractais. Processos multifractais apresentam além de dependência de longo prazo, diferentes leis de escala, sendo dessa forma mais abrangentes [3]. A dependência de longo prazo, presente em séries de tráfego e que tem importante impacto no desempenho de redes [4], pode ser constatada pelo decaimento lento da função de autocorrelação dessas séries temporais. Neste trabalho, utilizamos o conhecimento da função de autocorrelação de fluxos de tráfego multifractal em um esquema de alocação de banda para esses fluxos.

Algumas propostas de alocação de banda para fluxos de tráfego baseadas em predição realizadas por redes neurais e fuzzy merecem atenção pois mostram que o controle preventivo de tráfego pode ser bastante eficiente, uma vez que o mesmo tenta reduzir o congestionamento antes que este aconteça e se adapta bem às variações bruscas do tráfego de

Flávio Henrique Teles Vieira e Flávio Geraldo Coelho Rocha, Escola de Engenharia Elétrica e de Computação, Universidade Federal de Goiás. Lee Luan Ling, Scheila Guedes Garcez. Faculdade de Engenharia Elétrica e de Computação (FEEC), Universidade Estadual de Campinas. Emails: flavio@eeec.ufg.br, lee@decom.fee.unicamp.br, flavio.geraldo@gmail.com egarcez@dsif.fee.unicamp.br. redes [5][6]. Entre as propostas de controle de congestionamento que não dependem de mecanismos específicos de rede, destacamos o esquema apresentado por Adas et al. em [7], que usa o algoritmo adaptativo LMS[8] para a predição de tráfego de redes e estas predições são usadas como taxas exigidas pelos fluxos. Mais informações sobre o algoritmo LMS podem ser encontradas em [8]. Em [9], outro trabalho nesse sentido, os autores utilizam o algoritmo RLS que apresenta convergência mais rápida [10].

Algoritmos de controle adaptativos são mais adequados para aplicações multimídia em tempo real nas redes atuais devido ao processamento 'on-line' das informações [11]. Tendo em vista isso, apresentamos uma nova modelagem fuzzy com funções de base ortonormais (FBO) que através do algoritmo proposto ARFA (Agrupamento Regressivo Fuzzy Adaptativo) cria adaptativamente agrupamentos fuzzy à medida que dados de tráfego de entrada são disponibilizados, sendo capaz de prever eficientemente o tráfego de redes.

Neste trabalho, apresentamos um novo esquema de alocação de banda onde inserimos o preditor fuzzy proposto o qual faz uso de funções de base ortonormais calculadas a partir da função de autocorrelação do processo de tráfego baseada em um modelo multifractal.

A eficiência do esquema de alocação de banda proposto é avaliada através de comparações com outros esquemas em termos de taxa de perda, taxa média de ocupação do buffer e freqüência de sinalização.

O artigo está organizado da seguinte forma: na seção II, descrevemos as funções de base ortonormais de Laguerre. Na seção III, introduzimos alguns conceitos da análise multifractal e obtemos uma equação para o pólo utilizado nas funções de base ortonormais. Na seção IV, desenvolvemos um algoritmo de treinamento adaptativo para o modelo multifractal-fuzzy proposto e o comparamos com outros existentes na literatura. $\mathrm{Na}$ seção $\mathrm{V}$, empregamos o preditor proposto em um novo esquema de alocação de banda, verificando sua eficiência em relação a outros esquemas. Por fim, na seção VI, apresentamos as conclusões obtidas.

\section{FUNÇÕES DE BASE ORTONORMAIS}

Nesta seção, abordaremos alguns conceitos envolvendo funções de base ortonormais e sua relação com a modelagem fuzzy.

A base de Laguerre é usada em vários contextos de identificação e controle de sistemas não-lineares [12] [13]. 
Neste trabalho, adotamos a base de Laguerre especialmente porque esta é completamente parametrizada por um único pólo, o pólo de Laguerre $p$. O conjunto de funções de transferência associadas a esta base é dada pela seguinte equação:

$$
\Phi_{\text {mag }, j}\left(q^{-1}\right)=\sqrt{1-p^{2}} \frac{q^{-1}\left(q^{-1}-p\right)^{j-1}}{\left(1-p q^{-1}\right)^{j}}, \quad j=1, \ldots, n
$$

onde $p \in\{P:-1<p<1\}$ é o pólo das funções de Laguerre (base de Laguerre) e onde $q^{-j}$ é o operador de deslocamento. Pode-se notar que fazendo $p=0$ em (1), resulta na base $\Phi_{m a g, j}\left(q^{-1}\right)=q^{-j}$. Portanto, a base $\Phi_{m a g, j}\left(q^{-1}\right)=q^{-j}$ é um caso especial da base de Laguerre.

A saída de um modelo entrada-saída pode ser escrita como:

$$
y(k)=H\left(l_{1}(k), \ldots, l_{n}(k)\right),
$$

onde $\quad l_{j}(k)=\Phi_{m a g, j}\left(q^{-1}\right) u(k)$ é a j-ésima função de Laguerre no instante de tempo $k, n$ é o número de funções de base utilizadas, $u(k)$ é o sinal de entrada e $H$ é um operador não-linear. Note que a operação não-linear correspondente a $H$ pode ser realizada através da modelagem fuzzy.

As funções de Laguerre $l_{j}(k)$ são recursivas e podem ser obtidas por equações de estado da seguinte forma [12]:

$$
\begin{gathered}
\mathbf{l}(k+1)=A \mathbf{l}(k)+b \mathbf{u}(k) \\
y(k)=H(\mathbf{l}(k))
\end{gathered}
$$

onde $\mathbf{l}(k)=\left[l_{1}(k) \ldots l_{n}(k)\right]^{T}$. A matriz $A$ e o vetor $b$ dependem da ordem do modelo $n$ e do valor do pólo $p$ como segue [14]:

$$
\begin{gathered}
A=\left(\begin{array}{ccccc}
p & 0 & 0 & \ldots & 0 \\
1-p^{2} & p & 0 & \ldots & 0 \\
(-p)\left(1-p^{2}\right) & 1-p^{2} & p & \ldots & 0 \\
\vdots & \vdots & \vdots & \ddots & \vdots \\
(-p)^{n-2}\left(1-p^{2}\right) & (-p)^{n-3}\left(1-p^{2}\right) & (-p)^{n-4}\left(1-p^{2}\right) & \ldots & p
\end{array}\right) \\
b=\sqrt{\left(1-p^{2}\right)}\left[\begin{array}{lllll}
1 & -p & (-p)^{2} & \ldots & (-p)^{n-1}
\end{array}\right]^{T}
\end{gathered}
$$

O modelo não-linear representado pelas equações (3) e (4) consiste de um mapeamento linear entre a entrada $u(k)$ e as funções de Laguerre $l_{j}(k)$, mais o mapeamento entre $l_{j}(k) \mathrm{e}$ a saída do sistema $y(k)$. Obtém-se dessa forma, um modelo mais preciso do que o modelo NLMA (Non-Linear Moving Average) com o mesmo número de funções [14] [15].

Dado um número de funções de Laguerre $n$ (ordem do modelo), a estimação de um valor adequado para o pólo $p$ da base ortonormal resulta em uma melhor representação do sistema a ser modelado. Objetivamos introduzir informações a respeito do comportamento multifractal dos processos de tráfego no cálculo do pólo $p$. Assim, abordaremos o comportamento multifractal de processos estocásticos na próxima seção.

\section{ANÁlise MultifraCtAL}

\section{A. Tráfego Multifractal de Redes}

$\mathrm{Na}$ análise multifractal verifica-se o comportamento em escala de momentos estatísticos dos processos para estimar suas regularidades locais [16][17]. Através de ferramentas da análise multifractal algumas propriedades encontradas em processos reais podem ser verificadas. O tráfego de redes, por exemplo, ao ser considerado multifractal significa que possui uma estrutura de forte dependência inerente entre as amostras, com incidência de rajadas em várias escalas [17]. Estas características podem degradar o desempenho de rede em relação a fluxos de tráfego Gaussianos e de curta-dependência [18]. Definimos processos multifractais a seguir.

Definição 1: Um processo estocástico $X(t)$ é multifractal se satisfaz a equação:

$$
E\left(|X(t)|^{q}\right)=c(q) t^{\tau(q)+1}
$$

onde $t \in T$ e $q \in \mathrm{Q}, T$ e $Q$ são intervalos na reta real, $\tau(q)$ e $c(q)$ são funções com domínio $Q$. Normalmente, assumese que $T$ e $Q$ tenham comprimentos positivos, e que $0 \in T$, $[0,1] \subseteq Q$.

Os traços de tráfego reais apresentam suas propriedades multifractais caracterizadas pela função de escala $\tau(q)$ e o fator de momento $c(q)$, conforme se pode observar pela equação (7). Assim, um modelo multifractal deve capturar estas duas propriedades multifractais. Isto pode ser obtido pelo produto de uma cascata multiplicativa $\mu\left(\Delta t_{k}\right)$ e uma variável aleatória i.i.d positiva $Y$ em cada intervalo de tempo $\Delta t_{k}$ [19]. A variável $Y$ é independente da medida da cascata $\mu\left(\Delta t_{k}\right)$, então a série obtida denotada por $X\left(\Delta t_{k}\right)$ satisfaz a equação (7).

A função de escala $\tau_{0}(q)=\tau(q)+1$ pode ser precisamente modelada ao assumirmos que $R$ é uma variável aleatória em $[0,1]$ com distribuição beta simétrica $\operatorname{Beta}(\alpha, \alpha), \alpha>0$ [19]:

$$
\tau_{0}(q)=\log _{2} \frac{\Gamma(\alpha) \Gamma(2 \alpha+q)}{\Gamma(2 \alpha) \Gamma(\alpha+q)}
$$

onde $\Gamma($.$) corresponde à função Gama.$

Consideremos $Y$ como sendo uma variável aleatória cuja distribuição seja lognormal e definida pelos parâmetros $\rho$ e $\gamma$ e momento $E\left(Y^{q}\right)=e^{\rho q+\gamma^{2} q^{2} / 2}$. Assim sendo, um processo multifractal pode ser descrito por 3 parâmetros ( $\alpha$, $\rho, \gamma)$.

Os parâmetros $\alpha, \gamma$ e $\rho$ para um processo multifractal podem ser atualizados adaptativamente através dos valores 
das funções $\tau(q)$ e $c(q)$. Um método de estimação destas funções baseado na equação (7) é descrito em [20]

Seja o processo multifractal $X(n)$ discreto no tempo com parâmetros $\alpha, \rho$ e $\gamma$. A função de autocorrelação deste processo para os instantes de tempo $n$ e $k$, é dada pela seguinte equação [19]:

$$
E[X(n), X(n+k)]=e^{2 \rho+\gamma^{2}}\left(\frac{\alpha(\alpha+1)^{N-1}}{(\alpha+1 / 2)^{N}} k^{-\log _{2}\left(\frac{\alpha+1}{\alpha+1 / 2}\right)}\right) .
$$

onde $N=\log _{2}\left(N_{a}\right)$ e $N_{a}$ é a quantidade de amostras do processo.

\section{B. Pólo do Modelo Obtido a Partir da Função de Autocorrelação para Processos Multifractais}

Nesta seção, introduzimos uma expressão analítica para o pólo $p$ utilizado no cálculo das funções de base ortonormais.

Proposição 1: O pólo $p$ utilizado para o cálculo das funções de base ortonormais para o modelo multifractal-fuzzy pode ser dado por:

$$
p=-\frac{1}{\log _{2}\left(\frac{\alpha+1}{\alpha+1 / 2}\right)} .
$$

Prova: Ver Apêndice A

Note que uma vez que o pólo $p$ é dado em função do parâmetro multifractal $\alpha$, podemos estimá-lo adaptativamente.

\section{MOdElo MultiFraCtAL-FuZZY}

A estimação do valor do pólo permite que estendamos a interpolação fuzzy de modelos locais, que é a idéia central do modelo TSK [21], a um contexto de funções de base ortonormais. Nossa proposta se baseia em uma versão em espaço de estados do modelo TSK, ou seja, cada regra $R^{i}$ do modelo fuzzy representa um modelo de espaço de estados diferente:

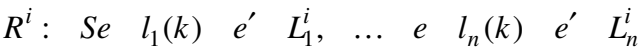

$$
\begin{aligned}
& \text { Então }\left\{\begin{array}{l}
\mathbf{l}_{i}(k+1)=A_{i} \mathbf{l}_{i}(k)+b_{i} \vec{x}(k) \\
y_{i}(k)=H_{i}\left(\mathbf{l}_{i}(k)\right)
\end{array}\right.
\end{aligned}
$$

onde a matriz $A_{i}$ e o vetor $b_{i}$ dependem do pólo $p_{i}(k)$ e $H_{i}\left(\mathbf{l}_{i}(k)\right)$ é o mapeamento que relaciona a saída $y_{i}(k)$ do modelo local $i$ a seu correspondente estado de Laguerre (funções de base ortonormais) $\mathbf{l}_{i}(k)=\left[\begin{array}{lllll}l_{1}(k) & l_{2}(k) & \ldots & l_{n}(k)\end{array}\right]$ , sendo $\vec{x}(k)=\left[\begin{array}{llll}x_{1}(k) & x_{2}(k) & \ldots & x_{n}(k)\end{array}\right]$ o vetor de entrada e $L_{j}^{i}$ a função de pertinência fuzzy para a regra $i$ associada a $j$-ésima variável de premissa. As variáveis de premissa são os estados de Laguerre do modelo multifractal-fuzzy resultante. A saída do modelo multifractal-fuzzy é dada por:

$$
y(k)=\frac{\sum_{i=1}^{C} y_{i}(k) w_{i}\left(\mathbf{l}_{i}(k)\right)}{\sum_{i=1}^{C} w_{i}\left(\mathbf{l}_{i}(k)\right)},
$$

onde $C$ é o número de regras (modelos locais) e os pesos $w_{i}\left(\mathbf{l}_{i}(k)\right)$ da regra $i$ são dados por:

$$
w_{i}\left(\mathbf{l}_{i}(k)\right)=\prod_{j=1}^{n} L_{j}^{i}\left(l_{j}(k)\right) .
$$

O modelo multifractal-fuzzy pode ser representado conforme as equações (3) e (4) e $H$ dado de acordo com as equações (12) e (13).

\section{A. Algoritmo de Treinamento Adaptativo para o Modelo multifractal-fuzzy}

Nessa seção, propomos um algoritmo de agrupamento regressivo fuzzy adaptativo (ARFA) para o modelo preditivo multifractal-fuzzy .

No algoritmo ARFA, levamos em conta a distribuição espacial dos dados considerando o erro de regressão e a distância entre os dados de entrada e os clusters. Seja a função custo do algoritmo ARFA definida como:

$$
J=\sum_{i=1}^{C} \sum_{k=1}^{N} u_{i k}^{2}\left(r_{i k} d_{i k}\right)^{2}
$$

sujeito a

$$
\sum_{i=1}^{C} u_{i k}=1, \quad \text { para } 1 \leq k \leq N
$$

onde $u_{i k}$ é o grau de ativação da $i$-ésima regra para o $k$ ésimo padrão de treinamento, $C$ é o número de regras fuzzy e $N$ é o número total de dados de treinamento. Na equação (14), $r_{i k}$ é o erro entre a $k$-ésima saída desejada $y(k)$ do sistema modelado e a saída da $i$-ésima regra $f_{i}\left(\vec{x}(k) ; \vec{a}^{i}(k)\right)$ com a $k$-ésima entrada, isto é,

$$
r_{i k}=y(k)-f_{i}\left(\vec{x}(k) ; \vec{a}^{i}(k)\right),
$$

com $i=1,2, \ldots, C$ e $k=1,2, \ldots, N$. Na mesma equação (14), $d_{i k}$ é a distância entre o vetor de entrada $\vec{x}(k)$ no instante discreto $k$ e o centro do $i$-ésimo cluster $\beta_{i}$, ou seja,

$$
d_{i k}=\vec{x}(k)-\beta_{i} .
$$

Seja $X \in \mathrm{P}^{N \times(n+1)}$ uma matriz onde seus elementos são os valores de $\vec{x}(k)$ em sua $j$-ésima coluna $j=1, \ldots, n+1$ (a primeira coluna de $X$ é toda composta por 1), $Y \in \mathrm{P}^{N}$ um vetor onde o $k$-ésimo elemento é o valor de $y(k)$ e $Q_{i} \in R^{N \times N}$ uma matriz diagonal onde a $k$-ésima diagonal é dada pelo termo $q(k)=u_{i k}^{2} d_{i k}^{2}$.

Como proposta, tomou-se como solução para este problema a aplicação de um algoritmo recursivo de estimação 
dos parâmetros representado pelas seguintes equações [22]:

$$
\begin{aligned}
& \vec{a}^{i}(k+1)=\vec{a}^{i}(k)+\left(P_{i}(k+1) x(k+1) q(k+1)\right) \times \\
& \times\left(y(k+1)-x(k+1)^{T} \vec{a}^{i}(k)\right)
\end{aligned}
$$

$\mathrm{e}$,

$$
P(k+1)=P(k)-\frac{q(k+1) P_{i}(k) x(k+1) x(k+1)^{T} P_{i}(k)}{1+q(k+1) x(k+1)^{T} P_{i}(k) x(k+1)},
$$

onde $x(k+1)$ é a $(k+1)$-ésima linha da matriz $X$ e $q(k+1)$ é o $(k+1)$-ésimo elemento da matriz diagonal $Q_{i}(k+1)$.

Para que se minimize a função custo (14), o grau de ativação $u_{i k}$ da $i$-ésima regra deve ser dado por:

$$
u_{i k}=\frac{1 / 2\left(r_{i k}^{2} d_{i k}^{2}\right)}{\sum_{i=1}^{C} 1 / 2\left(r_{i k}^{2} d_{i k}^{2}\right)} \text {. }
$$

Da mesma forma, encontra-se a seguinte equação para o centro do $i$-ésimo cluster $\left(\beta_{i}\right)$ :

$$
\beta_{i}=\frac{\sum_{z=1}^{N} r_{i z}^{2} u_{i z}^{2} \vec{x}(z)}{\sum_{z=1}^{N} r_{i z}^{2} u_{i z}^{2}}
$$

\section{EsTIMAÇÃo AdAPTATIVA DE BANDA UTILIZANDO O PREDITOR FUZZY PROPOSTO}

Os esquemas de alocação de banda para fluxos de tráfego de redes podem se beneficiar de algoritmos de predição da taxa de tráfego de modo a antecipar as ações para alocação de recursos e controle de congestionamento [23]. Neste sentido, o passo de predição pode ser ajustado de forma a possibilitar que a rede tenha tempo para obtenção e alocação dos recursos necessários.

Seja $A(\tau, t)$ um processo a tempo discreto correspondente ao tráfego acumulado (neste caso, número de bytes) no intervalo de tempo $(\tau, t)$ e que chega ao servidor para ser transmitido. Para satisfazer um limite de retardo $d_{r e q}$, qualquer pacote (ou dados) deve ser transmitido até o instante de tempo ' $t+d_{r e q}$ '. A banda $\varepsilon$, ou seja, a taxa necessária para atender a esse critério de retardo e para se ter perda nula de dados de tráfego, deve obedecer a seguinte relação:

$$
\mathcal{E}^{*}\left(t-\tau+d_{r e q}\right) \geq A(\tau, t)+b_{t-1} \quad \forall \quad t \geq \tau,
$$

onde $b_{t-1}$ corresponde ao número de bytes não enviados pela rede no instante anterior. A relação acima deve ser satisfeita para todo $t \geq 0$ e $\tau \geq 0$ onde $t \geq \tau$.

$\mathrm{O}$ procedimento que propomos para mapear o valor predito da intensidade de tráfego com a banda requerida é o seguinte: Seja $\triangle B W$ a quantia de banda finita e $C$ a máxima banda disponível. Determina-se o intervalo de banda $[(k) \Delta B W,(k+1) \Delta B W] \quad(k \geq 0)$, no qual o valor predito da taxa $P_{t+1}$ se encontra e usa-se o valor superior do intervalo $(k+1) \Delta B W$ ou a banda máxima disponível $C$ no caso de $C<(k+1) \Delta B W$ como banda requerida no instante $t+1$. Ou seja, a banda $B W_{t+1}$ no instante $t+1$ de acordo com a equação (22) é dada por

$$
B W_{t+1}=\min \left\{\left\langle\frac{\left(P_{t+1}+b_{t}\right) /\left(t+d_{r e q}\right)}{\Delta B W}\right\rangle \Delta B W, C\right\},
$$

onde o operador $\langle x\rangle$ representa o maior número inteiro mais próximo de $x$.

Como a banda requerida (23) é o valor superior do intervalo onde se encontra o valor predito da taxa, tem-se uma provisão de banda menos sensível às pequenas variações das predições, dentro dos intervalos de tamanho $\Delta B W$. Ao se aumentar o intervalo de banda, evita-se uma freqüente realocação de banda, reduzindo o custo de sinalização envolvido.

A. Avaliação de Desempenho do Esquema Proposto de Alocação de Banda

Utilizamos nas simulações, traços de tráfego TCP/IP (Transmission Control Protocol/ Internet Protocol) obtidos da Digital Equipment Corporation (DEC) ${ }^{1}$, traços de tráfego Ethernet obtidos da Bellcore ${ }^{2}$ e traços capturados na rede Petrobrás através de um analisador de dados DA350 da Acterna $^{T M}$, com uma resolução de 32 microsegundos [24].

Seja $z_{t}$ o valor observado de taxa de tráfego e $B W_{t}$ a banda fornecida no instante de tempo $t$. Comparamos o esquema de provisão de banda proposto com dois outros esquemas, entre eles, o esquema apresentado por Adas et al. [7], que usa o algoritmo adaptativo LMS [8] para a predição de tráfego de redes e estas predições são usadas como taxas exigidas pelos fluxos. Outro trabalho comparado [9], utiliza o algoritmo RLS que possui convergência mais rápida [10]. A fim de se estudar os efeitos do tamanho do intervalo de banda sobre todos os esquemas em questão, a equação (23) é aplicada nos 3 esquemas em comparação, onde a diferença entre eles consiste nos valores de $P_{t+1}$ dados pelos algoritmos de predição multifractal-fuzzy, RLS e LMS, respectivamente.

Os resultados de desempenho: taxa de perda e utilização para os 3 esquemas de alocação de banda aplicados à série de tráfego 10-7-S-1 da rede da Petrobrás são mostrados nas Figuras 1 e 2. Pode-se observar que o esquema de alocação de banda proposto atinge uma taxa de perda menor a uma frequiência de sinalização semelhante aos demais (Figura 3), porém a custo de uma utilização do enlace ligeiramente menor. Espera-se que isso ocorra quando é maior a precisão do algoritmo de predição utilizado. Pode-se concluir que a probabilidade de perda para determinado tamanho de buffer é

${ }^{1}$ http://ita.ee.lbl.gov/html/contrib/DEC-PKT.html

${ }^{2}$ http://ita.ee.lbl.gov/html/contrib/BC.html 
menor para o esquema de alocação de banda proposto (Figura 4).

\section{CONCLUSÃO}

As características dos fluxos de tráfego nas redes atuais como dependência de longo prazo e rajadas em múltiplas escalas tornam a modelagem e predição de tráfego tarefas difíceis e desafiadoras [25]. Neste trabalho, foi proposto um modelo multifractal-fuzzy cujo algoritmo de treinamento adaptativo permite que a predição tempo real do tráfego de redes, e por fim alocação mais precisa de banda, sejam realizadas com um número reduzido de regras nebulosas.

Uma das vantagens do esquema adaptativo de provisão de banda é que a alocação de banda pode ser realizada baseada nas amostras de tráfego disponíveis no instante de tempo atual. Como este esquema de provisão de banda se adapta segundo as mudanças do tráfego, um melhor aproveitamento dos recursos é obtido comparado a uma alocação estática de banda. O método de alocação de banda proposto consegue manter um bom equilíbrio entre perda de dados, freqüência de sinalização e utilização. Verificamos que o objetivo de se conseguir uma menor taxa de perda com a aplicação do nosso esquema preditivo de provisão de banda foi atingido. Tal resultado se deve a melhora de desempenho de predição proporcionada ao se utilizar o modelo multifractal-fuzzy. A partir do modelo de predição proposto, esquemas que considerem também minimização do custo de sinalização e diferentes valores de retardo de pacotes podem ser desenvolvidos. Questões estas que serão tratadas em trabalhos futuros.

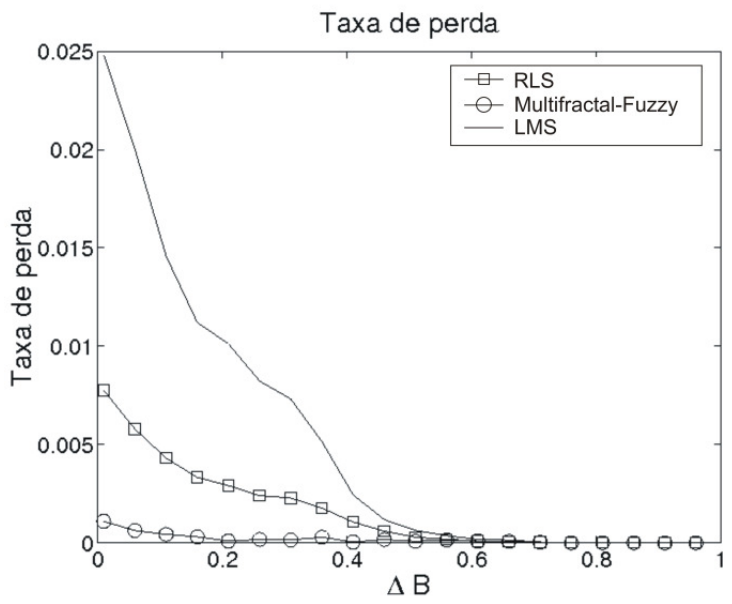

Fig. 1. Taxa de perda $\mathbf{x} \Delta B W$. Comparação de desempenho entre esquemas de alocação de banda

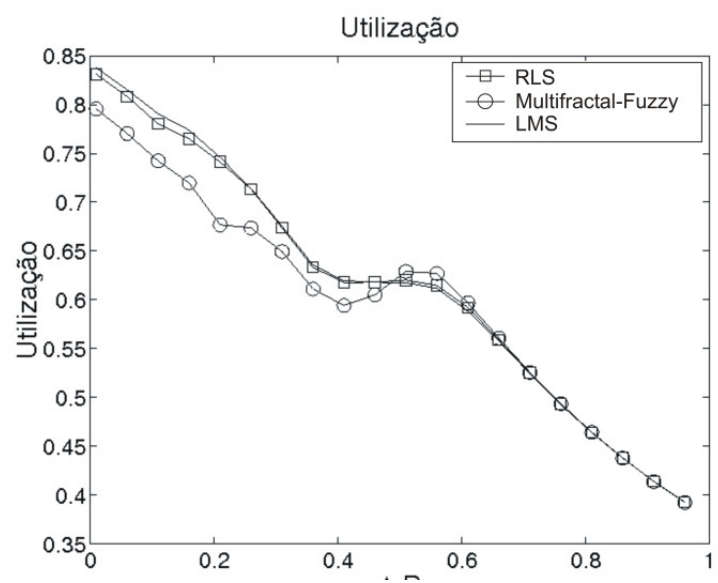

Fig. 2. Utilização $\mathbf{x} \Delta B W$. Comparação de desempenho entre esquemas de alocação de banda

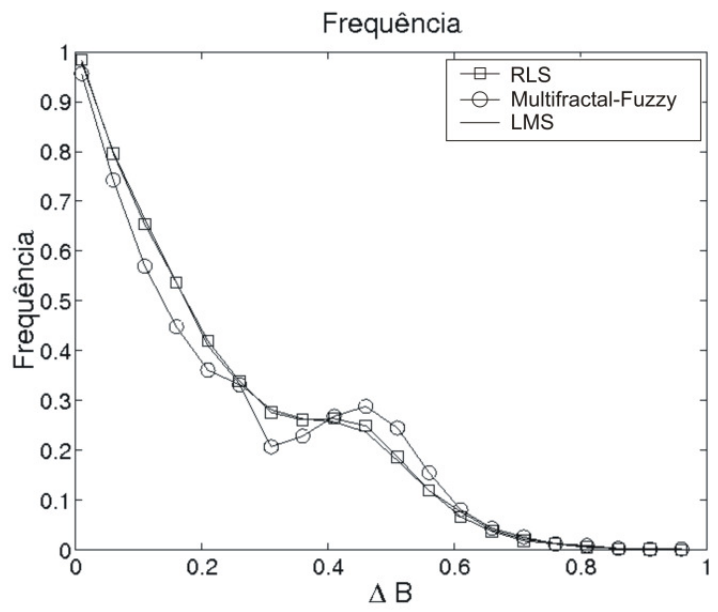

Fig. 3. Frequência de sinalização x $\Delta B W$

Função de distribuição acumulada complementar

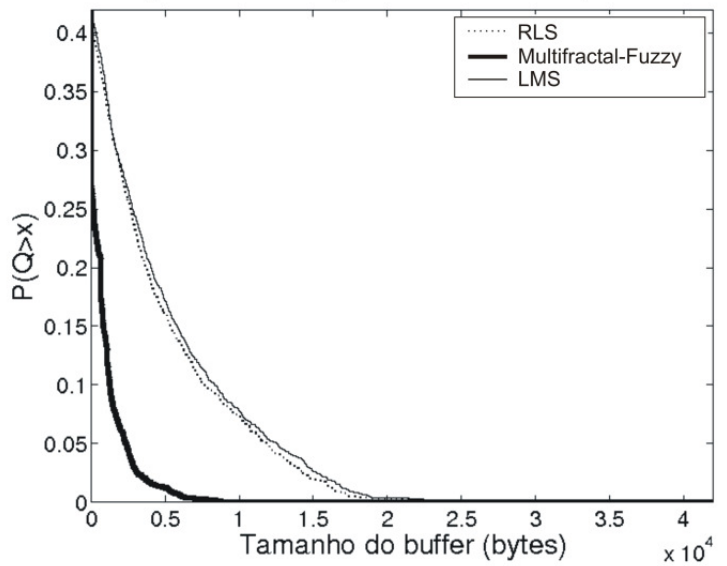

Fig. 4. $P(Q>x)$ x Tamanho do buffer. Análise do comportamento de fila dos esquemas de alocação de banda para o traço de tráfego 10-7-S-1 


\section{REFERÊNCIAS}

[1] S. Molnar and G. Terdik. "A general fractal model of internet traffic”. In IEEE LCN 2001, Tampa, Florida, Novembro 2001.

[2] M. S. Crouse e R. G.Baraniuk V. J. Ribeiro, R. H. Riedi. "Multiscale queueing analysis of long-range dependent traffic". Proc. IEEE INFOCOM, vol.2, pp. 1026-1035, Março 2000.

[3] I. W. C. Lee and A. O. Fapojuwo. "Stochastic processes for computer network traffic modelling". Computer Communications, vol. 29, pp.1-23, Março 2005.

[4] M. Grossglauser e J.-C. Bolot. "On the relevance of long-range dependence in network traffic". IEEE/ACM Transactions on Networking, vol. 7, no.5, pp.629-640, Outubro 1999.

[5] Y. C. Ouyang, C.-W. Yang e W. S. Lian. "Neural networks based variable bit rate traffic prediction for traffic control using multiple leaky bucket". Journal of High Speed Networks. vol. 15, no.2, pp.11-122, 2006.

[6] V. A. Aquino e J. A. Barria. "Multiresolution FIR neural-networkbased learning algorithm applied to network traffic prediction". IEEE Transactions on Systems, Man and Cybernetics-C, vol. 36 , no.2, pp.208-220, Março 2006.

[7] A. M. Adas. "Using adaptive linear prediction to support real-time VBR video under RCBR network service model". IEEE/ACM Trans. Net., vol. 6, no.5, pp.635-645, Outubro 1998.

[8] B. U. Toreyin, M. Trocan e A. E. Cetin. "LMS based adaptive prediction for scalable video coding". In ICASSP, Toulouse, França, 14 a 19 de Maio 2006

[9] S. Chong, S.-Q.Li e J. Ghosh. "Predictive dynamic bandwidth allocation for efficient transport of real-time VBR video over ATM". IEEE JSAC, vol. 13, no.1, pp.12-23, Jan. 1995.

[10] S. S. Haykin. Modern Filters. Macmillan Publishing Company, New York, 1989.

[11] H.-H Liu and P.-L. Hsu. "Design and simulation of adaptive fuzzy control on the traffic network". In International Joint Conference SICE-ICASE, pp. 4961-4966, Outubro 2006.

[12] G. H. C. Oliveira, R. J. G. B.Campello e W.C.Amaral. "Fuzzy models within orthonormal basis function framework". In IEEE International Fuzzy Systems Conference Proceedings, Seoul, Korea, 22-25 Agosto 1999.

[13] G. A. Dumont e Y. Fu. "Non-linear adaptive control via Laguerre expansion of Voltera kernels". Int. J. Adaptive Control and Signal Processing, vol. 7, pp.367-382, 1993.

[14] B. Ninness e F. Gustafsson. Orthonormal bases for system identification. In Proc. of 3rd European Control Conference, pp. 13-18, Roma, Itália, Setembro 1995.

[15] B. Wahlberg e L. Ljung. "Hard frequency-domain model error bounds from least-squares like identification techniques". IEEE Trans. on Automatic Control, vol. 37, no.7, pp.900-912, Julho 1992.

[16] A. Feldmann, A. C. Gilbert e W. Willinger. "Data networks as cascades: Investigating the multifractal nature of Internet WAN traffic". pp. 25-38. ACM/SIGCOMM'98, Vancouver, 1998.

[17] R. H. Riedi, M. S. Crouse, V. J. Ribeiro e R. G. Baraniuk. "A multifractal wavelet model with application to network traffic". IEEE Trans. on Information Theory, vol. 45, no.3, pp. 992-1018, Abril 1999.

[18] K.Park e W. Willinger. Self-similar Network Traffic and Performance Evaluation. John Wiley and Sons, New York, 2000.

[19] T. D. Dang., S. Molnar e I. Maricza. "Capturing the complete characteristics of multifractal network traffic". In Globecom 2002 Taipei, Taiwan, Novembro 2002.
[20] T. D. Dang; S. Molnar e I. Maricza. "Queuing performance estimation for general multifractal traffic". Int. J. Commun. Syst., vol. 16, no.2, pp.117-136, 2003

[21] T. Takagi e M. Sugeno. "Fuzzy identification of systems and its applications to modeling and control". IEEE Trans. Syst., Man, Cybern., vol. 15, pp.116-132, Jan. 1985

[22] P. Young. Recursive Estimation and Time Series Analysis: An Introduction. Communications and Control Engineering Series. Springer-Verlag, New York, 1984.

[23] H. T. Tran e T. Ziegler. "Adaptive bandwidth provisioning with explicit respect to QoS requirements". Computer Communications, vol. 28, pp.1862-1876, Janeiro 2005.

[24] Lee Luan Ling et al. A computational tool and optimization methods for multimedia traffic characterization and effective bandwidth estimation on modern communication networks. Relatório Técnico. Projeto Ericson UNI-20, Laboratório de Reconhecimento de Padrões e Redes de Comunicações (LRPRC) -Unicamp, Março 2002.

[25] G. R. Bianchi, F. H. T. Vieira e L.L.Ling . Predictive Dynamic Bandwidth Allocation Based on Multifractal Traffic Characteristics. Lecture Notes in Computer Science, v. 3126, p. 21-30, 2004.

\section{APÊNDICE A}

Seja $X(t)$ um processo cuja função de autocorrelação é representada por $r_{x}(k)=E[X(t+k) X(t)]$ e $\Gamma_{j+1} \quad(j=1, \ldots, n)$ , o coeficiente de reflexão para encontrar um modelo AR (AutoRegressivo) de ordem $n$ [10]. Os valores dos coeficientes $a_{n+1}(j)$ do modelo AR podem ser calculados a partir de $a_{n}(j)$ de tal forma que [10]:

$$
r_{x}(k)+\sum_{l=1}^{p} a_{p}(l) r_{x}(k-l)=0 ; \quad k=1,2, \ldots, p
$$

Assim, o pólo de um modelo com $(j=1)$ é dado por [10]:

$$
\Gamma_{j+1}=-\frac{\gamma_{j}}{\varepsilon_{j}}
$$

onde $\varepsilon_{j+1}$ é o erro de modelagem.

Inserindo a equação de autocorrelação (9) em (25), e sabendo que $p=\Gamma_{1}$, temos a seguinte equação para o pólo $p$ :

$$
p=-\frac{1}{2^{\log _{2}\left(\frac{\alpha+1}{\alpha+1 / 2}\right)}}
$$

